\title{
Special issue on real-time 3D imaging and processing
}

\author{
Sen Wang
}

Published online: 17 January 2012

(C) Springer-Verlag 2012

In recent years, with the development of 3D techniques in computer vision and graphics, 3D imaging and processing has considerably grown leading to a number of challenges including performing real-time 3D data acquisition, representation, processing, editing, compression, transmission, optimization and visualization, etc. Real-time 3D imaging and processing systems demand a trade-off between accuracy and computational complexity. Although 3D imaging systems are available to deliver high spatial resolution for real-time recording, real-time analysis is often the bottleneck to enable the entire processing chain working in real-time.

On the other hand, in order to cope with real-time 3D imaging and processing challenges, some motion capture systems use a small set of tracking points with a fairly high temporal resolution. In addition, recent advances in 3D imaging systems, coupled with major advances in $3 \mathrm{D}$ applications such as 3D entertainments including 3D TVs, 3D internet, games and movies, have led to dramatic increases in the variety and amount of 3D data processing over diverse media. The explosive growth of this technology necessitates more research into algorithms that can offer 3D imaging and processing for these rapidly evolving applications which demand real-time operations.

This special issue on Real-Time 3D Imaging and Processing is intended to incorporate contributions to the topic in theory and application that encapsulate recent developments in real-time capture, representation, and processing algorithms as well as applied systems. The issue consists of

S. Wang $(\bowtie)$

Kodak Research Laboratories, Eastman Kodak Company, 1999 Lake Ave, Rochester, NY 14650, USA

e-mail: sen.wang@kodak.com five papers and the remainder of this editorial presents a brief summary for each of them.

In the paper "Exploration of 3D grid caching strategies for ray shooting", Mancini et al. present a new "nD-AP" Cache ( $n$-Dimensional Adaptive and Predictive Cache) architecture aiming at efficient data access for 3D grid ray shooting. A theoretical model of the 3D version of the cache is setup in order to predict the cache efficiency for given statistical characteristics of the access sequences and for given parameters of the cache. Further improvement and applications of the $\mathrm{nD}-\mathrm{AP}$ Cache are discussed and comparisons with standard caches show that the nD-AP Cache is two times more efficient than an associative cache and with four times less memory.

In the paper "Efficient FPGA implementation of homodyne-based time of flight range imaging", Jongenelen et al. describe an efficient real-time FPGA algorithm for determining phase and distance in time of flight range imaging systems. The scarcest resource in this implementation is RAM, and an analysis is presented to maximize the efficiency of this resource whilst maintaining acceptable processing accuracy. The algorithm can be extended for processing multiple simultaneous modulation frequencies. An efficient method to determine unambiguous range is also discussed.

In the paper "Real-time detection of colored objects in multiple camera streams with off-the-shelf hardware components", Lampert and Peters propose a high speed vision system-Rtblob that can detect objects in cluttered scenes based on their color and shape at a speed of over 800 frames per second. Because the system is available as open-source software and relies only on off-the-shelf PC hardware components, it can provide the basis for multiple application scenarios such as in a robotic table tennis scenario to estimate ball trajectories through $3 \mathrm{D}$ space 
simultaneously from 4 cameras images at a speed of $200 \mathrm{~Hz}$.

In the paper "Real-time and low-power processing of 3D direct/inverse discrete cosine transform for low complexity video codec", Saponara addresses the problem of real-time and low-power 3D DCT/IDCT processing by presenting a context-aware fast transform algorithm and a family of VLSI architectures characterized by different levels of parallelism. Implemented in submicron CMOS technology, the proposed hardware macro-cells support the real-time processing of main video formats with different trade-offs between circuit complexity, power consumption and computational throughput.

In the paper "High-resolution, real-time 3-D imaging with fringe analysis", Karpinsky and Zhang present Realtime 3D imaging systems based on structured lighting and fringe analysis. They explain the principles behind fringe analysis-based techniques and provide experimental results from systems using these techniques.

To conclude, I would like to thank the authors, who contributed to this special issue by reporting about their research and findings on the topic tackled in this issue, all of them reporting their experiences and tracking the path for future research issues and challenges. I would also like to thank the anonymous referees who played a key role in the review and selection process with their insightful comments, ensuring the Special Issue includes only the submissions of highest technical quality.

\section{Author Biography}

Dr. Sen Wang is a senior research scientist in Kodak Research Laboratories, Eastman Kodak Company. He received his Ph.D. degree in Computer Science from Stony Brook University in 2008 and a M.Sc. degree in 2006. He also received a M.E. degree from Chinese Academy of Science in 2003 and a B.S. degree from Shandong University in 2000. Currently, Dr. Wang serves as an associate editor for the Journal of Mathematical Imaging and Vision. $\mathrm{He}$ is also on the editorial board of Journal of Real-time Image Processing and Journal of Personal and Ubiquitous Computing. His main interests are Computer Vision, Computer Graphics, Stereo/3D Imaging, Biometrics, Image/Video Processing, and Human Computer Interaction. Dr. Wang has more than 20 research papers and 30 patent applications in the above areas. 